\title{
Combining Multiple one-class Classifiers for Anomaly based Face Spoofing Attack Detection
}

\author{
Soroush Fatemifar, Muhammad Awais, Shervin Rahimzadeh Arashloo ${ }^{\star}$, Josef Kittler \\ Centre for Vision, Speech and Signal Processing, University of Surrey, UK \\ ${ }^{\star}$ Bilkent University, Turkey \\ \{s.fatemifar, m.a.rana, s.rahimzadeh, j.kittler\} @surrey.ac.uk
}

\begin{abstract}
One-class spoofing detection approaches have been an effective alternative to the two-class learners in the face presentation attack detection particularly in unseen attack scenarios. We propose an ensemble based anomaly detection approach applicable to one-class classifiers. A new score normalisation method is proposed to normalise the output of individual outlier detectors before fusion. To comply with the accuracy and diversity objectives for the component classifiers, three different strategies are utilised to build a pool of anomaly experts. To boost the performance, we also make use of the client-specific information both in the design of individual experts as well as in setting a distinct threshold for each client. We carry out extensive experiments on three face anti-spoofing datasets and show that the proposed ensemble approaches are comparable superior to the techniques based on the two-class formulation or class-independent settings. *
\end{abstract}

\section{Introduction}

Although biometric systems have made a remarkable progress in recent years, they are potentially vulnerable to face spoofing attacks, especially to unseen types of presentation attacks (PA). A spoofing attack occurs when an impostor tries to fool a face recognition system by using PAs to authenticate himself/herself as a genuine client. The most common face PAs in practice include print attack, replay attack, and 3D masks. To prevent spoofing attacks, face recognition systems should be equipped with highly capable countermeasures to reduce the security concerns of unknown attack attempts.

To counteract PAs, the majority of approaches in the

* This work was supported in part by the EPSRC Programme Grant (FACER2VM) EP/N007743/1 and the EPSRC/dstl/MURI project EP/R018456/1.

978-1-7281-3640-0/19/\$31.00 @ 2019 IEEE literature formulate the face spoofing problem as a binary classification task in which a spoofing detector tries to learn distinguishable features separating genuine accesses from spoofing attacks [24]. However, the two-class formulation has shown some drawbacks in the real-world spoofing scenarios[15]. To address this drawback, anomaly detection based models have been proposed in recent studies $[3,15]$ as alternative spoofing detection systems. To construct anomaly classifiers, researchers have considered three broad categories: (i) Learning with positive samples only (ii) Learning with positive samples and small proportion of negative samples (iii) Learning with positive and unlabelled data. In this paper, we adopt the same anomaly detection approach in $[3,15]$ by building spoofing detectors using only genuine accesses data, corresponding to category (i).

The majority of existing studies in the spoofing attack detection assume that the relevant information is independent of the class identity. However, it is reasonable to argue that anti-spoofing systems are likely to be more successful if specific biometric traits of individual clients are considered. The benefit of using client-specific information has been first recognised in [8].However, the idea was investigated in the context of a two-class anomaly detection model, and this requires access to all variations of spoofing attacks in the enrolment stage, which is unrealistic in practical scenarios. To overcome this limitation, authors in [15] proposed a way to use client-specific information to train anomaly classifiers by using only genuine accesses. In this paper, we investigate the merits of using client-specific information for fusion of anomaly classifiers and setting a subject-specific decision threshold for them.

In the quest to increase the generalisation capacity of spoofing detectors, a number of authors have proposed ensemble techniques to improve the spoofing detection performance by combining the decisions of individual classifiers $[2,22]$. An ensemble of classifiers thrives if individual members are accurate and diverse. In this paper, three approaches are adopted to create POC. They include oneclass Support Vector Machines (SVM), Gaussian Mixture 
Models (GMM), and Mahalanobis Distance outlier detector (MD) which have all shown to achieve a remarkable degree of success in spoofing detection [3]. Next, seven different regions of the face are considered to inject diversity in the performance of one-class classifier designs. Third, motivated by the promising results achieved by deep Convolutional Neural Networks (CNN) [38, 29, 22] compared to traditional feature extraction methods such as LBP[26] and BSIF[18], our anomaly classifiers are trained using the $\mathrm{CNN}$ representations of face data as input.

Some ensemble techniques for combining one-class classifiers have been studied outside the field of biometrics $[4,19]$, but never applied to the spoofing detection problem. Our aim is to redress this issue by proposing a simple fusion method based on a novel score normalisation strategy, which is particularly relevant for biometrics, as it allows a consistent control of false rejection rates. We combine oneclass classifiers in a pool to improve the accuracy of spoofing attack detection. In previous studies, even if the component classifiers are designed using genuine access data only, their fusion in an ensemble is facilitated with the help of spoofing attack samples [4]. We develop a fusion method based on weighted averaging of component classifiers using Genetic Algorithms (GA). The GA is a very popular optimisation method due to its simplicity, minimal requirements, ease of parallelism, and robustness. Although GA fusion approach is very effective, it compromises the philosophy of anomaly detection. In order to adhere fully to the spirit of anomaly detection, we also propose a fusion method for combining one-class spoofing attack detectors, which is trained on genuine access samples only.

We evaluate the proposed solutions on the existing antispoofing datasets, namely Replay-Attack [7] and ReplayMobile [10] to make a fair comparison with the state of the art studies. We also perform experiments on the ROSEYoutu [21] dataset, which is more challenging, as it contains spoof samples of higher resolution and imaging quality, covering a diverse variety of illumination conditions, camera sources, and a wider spectrum of attack categories. We advocate the same evaluation protocol proposed in [15] to implement a client-specific variant of the anomaly detection approach in the ROSE-Youtu dataset.

In summary, the main contributions of this paper are as follows: a) We develop a solution for the face spoofing detection problem by fusing multiple anomaly experts. A pool of 63 learners has been generated by creating different combination from the set of three anomaly detectors, seven regions of face and three $\mathrm{CNN}$ architectures. b) We propose a novel normalisation approach to support a multiple spoofing detector fusion using normal data only. c) We adopt a clientspecific approach for the design of both, individual spoofing attack detectors as well as ensemble models. d) By means of extensive experiments, we demonstrate that the proposed subject-specific anomaly detector fusion achieves superior performance compared to other methods.

\section{Ensemble of spoofing attack detection ex- perts}

The key prerequisite of classifier fusion is to create a POC, in our case a pool of face spoofing attack detectors, that are diverse and have the capacity to provide complementary information to enhance the recognition performance. There are different strategies to construct a POC. They include i) training set manipulation (bagging, boosting), ii) the use of different classifier models, iii) alternative classifier model parameterisations, iv) use of complementary data modalities, v) resorting to different subspaces. The aim of this paper is not to conduct an exhaustive exploration of different designs. Rather, the goal is to investigate the potential of multiple one-class classifier fusion. In this context we created our pool using simple mechanisms which can broadly be classified into the categories ii) and v) above. In particular, we use different regions of the face to define different subspaces, and different anomaly detection models. We also inject diversity by using different features extracted from the input data. Here we limit ourselves to deep neural network features obtained using different pretrained CNNs. The combination of a particular region, feature representation and classification model produces one expert for the ensemble.

The different mechanisms used are described in the following Subsections. In Subsection 2.1 we define the various facial regions providing an input to a spoofing attack detector. These regions are fed into pretrained neural networks described in Subsection 2.2 to produce different features for the anomaly detection process. Subsection 2.3 introduces the one-class classifiers selected for our study.

\subsection{Facial Regions}

Different parts of the face present distinct input information for decision making and by definition introduce diversity. At the same time, as different regions of the face exhibit disparate degree of appearance variation induced by changing expressions and talking face dynamics, selecting different regions provides the opportunity to identify facial parts that have the propensity to convey spoofing anomalies in a more reliable manner. Figure 1 illustrates the way in which the face image is divided into seven different regions. As can be seen in Figure 1, the majority of the face regions contain the eyes which have been extensively studied in spoofing detection. To extract the original face from a video frame, first a given frame is cropped according to its corresponding face bounding box which is provided as part of the dataset annotation. The original image is cropped by a tighter bounding box to minimise the effect of background on the spoofing detection performance. This process leads 


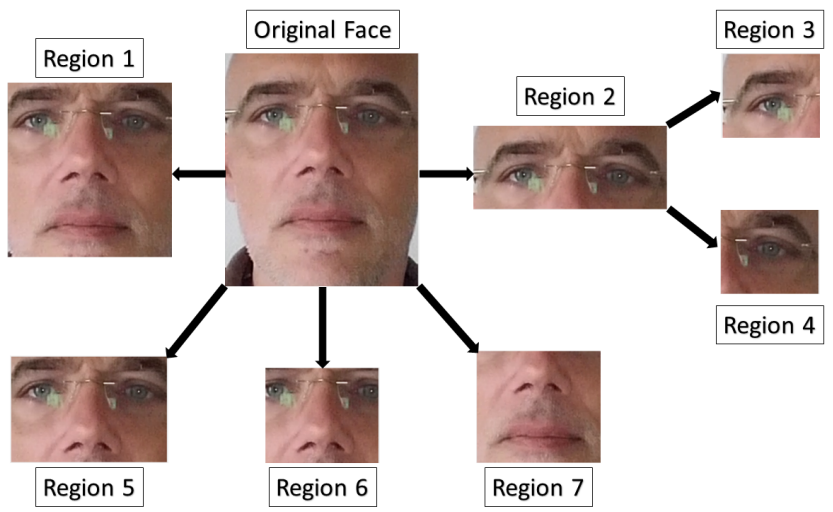

Figure 1. The original face is divided to seven different regions.

to the construction of Region 1 which serves as a new template to extract the remaining six facial regions. To assess the merits of the eyes region which has been investigated in liveness based spoofing attack detection [33], both eyes, right eye and left eye regions are extracted to form Regions 2,3 , and 4 respectively. Region 5 and Region 6 focus on the nose part of the face which has been studied extensively in the landmark detection applications [30]. Finally, Region 7 is adopted to capture the mouth and lips.

\subsection{Access Data Representation}

The gamut of image data representations used for face spoofing detection is very extensive. They include LBPs, LPQs [27], and image quality features [16]. Their relative effectiveness has been investigated in [3]. With the advent of deep learning, the recent research has also looked at the potential of features extracted by deep neural networks [13]. The motivation for investigating CNN features was to establish whether face spoofing detectors could use the same representation as face matching. In [15] it has been found that $\mathrm{CNN}$ features are very powerful. In addition, their adoption could greatly simplify the design of biometric systems. Here we adhere to the same philosophy and limit our study to CNN features only. As in [15], we consider CNN features extracted by networks such as GoogleNet [32], ResNet50 [17] and VGG-verydeep-16 (VD16) [31] tuned to extract powerful representation for face matching, as well as features output by $\mathrm{CNN}$ networks pretrained for other tasks.

\subsection{Anomaly Classifiers}

Anomaly detection is the identification of unusual data points deviating from the behaviour of the majority of the data. To construct an anomaly detector in spoofing scenarios, only real data can be used to train one-class classifiers. Spoofing samples are only considered in the evaluation stage. This paper adheres to the same anomaly detection philosophy according to which one-class classifiers are trained on genuine access data only. The anomaly detectors used in this paper are as follows:

One-CLASS SVM: Support Vector Data Description (SVDD) [34] is a one-class extension of SVM. SVDD encloses the normal training data by a minimum radius hypersphere. Outliers of the model can be flagged as the test samples falling outside the hypershpere.

One-CLASS MD: Assuming that genuine access data follows a single-mode Gaussian distribution, the Mahalanobis distance of a test sample to the mean can serve as an output of a one-class MD spoofing detector.

One-CLASS GMM: A Gaussian mixture model is a parametric probability density function defined as a weighted sum of Gaussian component densities. Its model parameters are estimated using the Expectation Maximization algorithm [11]. Outliers are detected by measuring the minimum MD to the respective mixture components.

\subsubsection{Client-Specific versus Client Independent Anomaly Detection}

The principal assumption to formulate spoofing detection problem with one-class classification scheme is that spoofing attacks would generate scores deviating from normal scores, and could be detected as outliers of the normal scores distribution. To this end, a threshold should be set at a predefined level of confidence to reject a given proportion (usually 1-15\%) of normal scores. If a development set was available, this can be done by predetermining a threshold so that the False Rejection Rate (FRR) and False Acceptance Rate (FAR) are balanced, which produces an equal error rate (EER). In the anomaly formulation adopted here the Half Total Error Rate (HTER) would correspond to the operation point defined by the selected level of confidence. In the majority of cases, class-independent thresholds fail to perform effectively because when the data of various clients are merged into a single population in an ensemble, it becomes much harder to find a single threshold that can be applied to various clients jointly. This limitation is depicted in Figure 2. To address this problem, first, we combine classification scores of POC spoofing detectors categorised by client specific information of each subject. Next, we set a client specific threshold for each population of scores accordingly.

\section{Fusion of anomaly detection experts}

\subsection{Literature}

To date, the investigation of the merits of fusing anomaly classifiers has been frequently confined to other applications rather than biometrics. To combine one-class classifiers, authors in [35] argued that if their outputs are standardised first, then it is possible to use traditional combination rules such as mean vote, mean weighted vote, prod- 

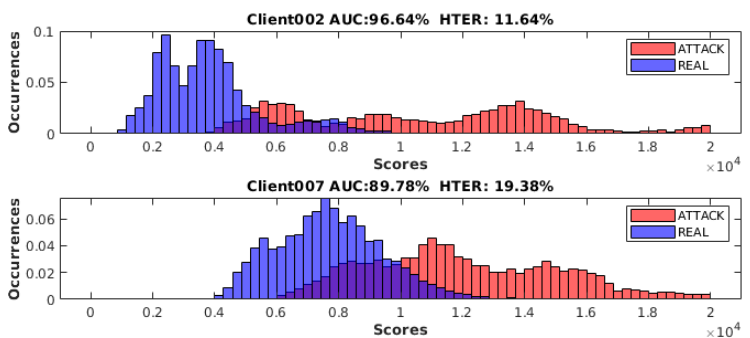

Figure 2. Score distributions of two clients from Replay-Mobile dataset using MD classifier and GoogleNet [32] features.

uct of the weighted votes, mean of the estimated probabilities, and product combination of the estimated probabilities for classifier fusion. The authors in [20] demonstrated the merits of using SVDDs fusion for image database retrieval which leads to improved retrieval precision. In another work [25], the authors combined several anomaly classifiers using the random subspace approach for the purpose of online signature verification, leading to a reduction of error rate. The authors in [5] proposed fusion of one-class SVMs for biometric applications. They used z-score, minmax, and column norm normalisation approaches to combine classification outputs. They experimentally concluded that min-max normalisation with weighted sum gives the best result. Another work [12] proposed an ensemble of one-class fuzzy KNN classifiers optimised by genetic algorithms to produce fused similarity measures. They showed that with the optimal parameters, the overall recognition rate improves in the majority of cases.

It cannot be overemphasised that when we have only normal access data for training, there are not many options available for expert fusion. We cannot even consider weighted averaging, as it is difficult to define an objective function that could be optimised to find the best mixing of scores. This situation is different when some samples from the negative distribution are available. Such a scenario will be discussed in Section 3.3. First we discuss simple averaging in Section 3.3 and show its expected benefits. A prerequisite is an effective score normalisation targeted for the spoofing attack detection application, which is the subject of the next Subsection.

\subsection{Confidence level score normalisation}

Let us consider $K$ anomaly detection experts, each producing score $s_{i}, i=1, \ldots, K$. Score $s_{i}$ represents a measure of affinity of an observation with the $i^{t h}$ outlier detection model built using a set of training observations, all corresponding to normal accesses. We conjecture that it should be possible to benefit from the multiple expert opinions by combining them using a very simple fusion rule, namely the sum rule. As the scores of different experts may be quite disparate in terms of their range of values, before applying the simple fusion rule, they have to be normalised. We could opt for a variety of normalisation procedures, such as scaling by $\max$ - min, a zero mean - unit variance normalisation, etc. In order to be consistent with the spirit of outlier detection, we shall normalise the scores by scaling so that for each expert a selected confidence level threshold $\rho$ maps to unity. Thus for each expert the proportion of normal samples lying under the tail of the score distribution above point 1 equals $1-\rho$. Let the confidence level cut off point for the distribution of scores $s_{i}$ be $s_{i}(\rho)$. The normalised score $\tilde{s}_{i}$ is then given by

$$
\tilde{s}_{i}=\frac{s_{i}}{s_{i}(\rho)}
$$

Note that for a $100 \%$ confidence level, the proposed score normalisation will be the same as dividing by the maximum score. For different confidence levels the normalisation factor in the denominator will differ.

\subsection{Fusion by Averaging}

In the simple averaging fusion, which does not require any attack data for training, the final prediction of the given sample of $x$, denoted by $H(x)$, is obtained by averaging the outputs of individual classifiers directly as follows:

$$
H(x)=\frac{1}{K} \sum_{i=1}^{K} \tilde{s}_{i}
$$

To reflect the relative importance of individual experts, specific weights $w_{i}$ may be assigned to the respective component classifiers. The combined output of weighted averaging is obtained as follows:

$$
H(x)=\frac{1}{K} \sum_{i=1}^{K} w_{i} \tilde{s}_{i}
$$

However, to train a weighted averaging system, some attack samples are required. In the present work, GA is adopted for computing the optimum weights in eq. (3). In GA, designing chromosome and fitness function plays an important role since they affect the complexity of the optimisation problem. As depicted in Figure 3, a novel encoding strategy is proposed to design a chromosome for the GA optimisation. It reflects the importance of each $\mathrm{CNN}$, classifier, and region for the spoofing attack detection task. Each chromosome translates into a fused score via an exponential function defined as

$$
H(x)=\frac{1}{R K N} \sum_{r=1}^{R} \sum_{l=1}^{L} \sum_{n=1}^{N} 2^{\left(w_{r}^{R}+w_{k}^{K}+w_{n}^{N}\right)} \tilde{s}_{r, l, n}
$$

where $\tilde{s}_{r, l, n}$ is the score for the given frame $x$ obtained from the $r$-th facial region, $l$-th anomaly classifier, and $n$ th CNN. The exponential weights rather than linear ones are applied to magnify the differences among the weights and to 
speed up the computation. The objective of the fitness function is to select those chromosomes achieving the lowest HTERs with the fused scores $H(x)$. Both, genuine access and attack data are used for the optimisation. According to

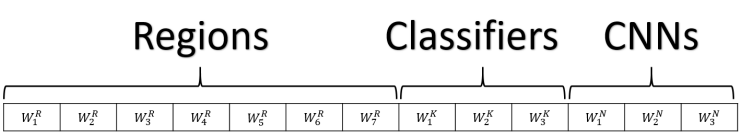

Figure 3. The proposed chromosome structure containing 13 genes that includes seven regions, three classifiers and three CNNs.

Figure 3, the first $\mathrm{R}=7$ genes convey the importance of the different regions. The next $\mathrm{L}=3$ genes represent the weighting of the anomaly experts while the last $\mathrm{N}=3$ genes designate the significance of each CNN. To initialise the weights in the first step of GA, a random integer value between zero and ten is assigned to each gene. Consequently, an objective of the fitness function in this paper is to minimise the HTER.

\section{Experiments}

The aim of the experiments described in this section is to evaluate the proposed fusion solutions and compare them with other one-class and multiclass classification approaches. Experiments are performed on three benchmark anti-spoofing datasets. Details regarding the implementation and the experimental protocols are discussed in the subsequent sections.

\subsection{Datasets and Protocols}

Three public face spoofing datasets, namely ReplayAttack, Replay-Mobile, and Rose-Youtu are used in our experiments. Replay-Attack contains 1300 videos with lowresolution while Replay-Mobile consists of 1200 videos with high-resolution. ROSE-Youtu is a comprehensive antispoofing dataset with 3350 videos covering a large variety of illumination conditions, camera models, and attack types. As noted earlier, to be consistent with the spirit of the anomaly detection formulation, only real-access data is used to build both class-specific and class-independent approaches. For Replay-Attack and Replay-Mobile datasets, the enrolment set available for each client is used for training the class-specific and class-independent approaches. Since enrolment set is not included in the Rose-Youtu dataset, we follow the evaluation protocol in [15] to implement the class-specific approaches.

\subsection{Implementation Details}

Before feeding video sequences to the pre-trained CNNs, each frame is photometrically normalised using the retina method [37] to reduce the negative impact of different lighting conditions. For the Rose-Youtu dataset, face bounding
Table 1. The HTERs of two different client of Replay-Mobile dataset based on two scenarios.

\begin{tabular}{|c|c|c|c|c|c|}
\hline \multirow{2}{*}{} & \multicolumn{2}{|c|}{ Client ID 4 } & \multicolumn{3}{c|}{ Client ID 19 } \\
\cline { 2 - 6 } & \multicolumn{2}{|c|}{ GoogleNet } & \multicolumn{3}{c|}{ Region 1 } \\
\cline { 2 - 6 } & Region 1 & Region 7 & GoogleNet & ResNet50 & VD16 \\
\hline MAH-Spec & 26.15 & 11.94 & 4.03 & 4.25 & 4.22 \\
\hline GMM-Spec & 32.33 & 13.77 & 4.54 & 3.12 & 1.35 \\
\hline SVM-Spec & 40.77 & 14.04 & 16.26 & 4.54 & 1.09 \\
\hline
\end{tabular}

boxes are detected by the Viola-Jones algorithm [36]. Features of the pre-ultimate layer of each network serve as an input to the anomaly detectors. The one-class SVM classifier implementation is based on the SVDD classifier from the LIBSVM [9]. In the case of the MD model, the parameters of the Gaussian distribution of the feature vector are estimated using the training data. For each test sample, the Mahalanobis distance is computed as a spoofing detection score. For the GMM classifier, the minimum Mahalanobis distance of the test sample from all $\mathrm{K}$ components is regarded as the spoofing detection score. The value of $\mathrm{K}$ is set for each dataset based on experimental optimisation. To use GA in the proposed weighted averaging fusion, we set the value of the initialisation population, crossover rate, and mutation rate to $20,0.4$, and 0.1 , respectively.

\subsection{Results}

Extensive experiments are carried out in this section to compare the performance of the proposed ensemble techniques with traditional single classification systems. In Table 1, two different settings are used to demonstrate the merits of using different class-specific classifiers, networks, and face regions in the fusion. According to Table 1, HTERs of the particular client (ID=4) in the Replay-Mobile dataset are reported according to the representations of GoogleNet for Region 1 and Region 7. Surprisingly, Region 7 outperforms Region 1, which corresponds to the whole image, by more than $15 \%$ in terms of HTER. For the other client $(\mathrm{ID}=19)$, the representation provided by GoogleNet is the best for Class-specific MAH while VD16 is the best CNN for GMM and SVM.

In Table 2, the performance of the individually best class-specific and class-independent classifiers is reported. According to Table 2, all class-specific anomaly detectors outperform class-independent ones significantly. It is interesting that in Replay-Attack, all variations of class-specific detectors are almost five times better than class-independent ones. For the Replay-Mobile and Rose-Youtu, there is still a considerable gain of roughly $3 \%$ in using the class-specific settings. Table 3 compares the HTERs performance of single class-specific anomaly classifiers against the proposed ensemble solutions. According to Table 3, the proposed simple averaging fusion is superior to their individual members by around $1 \%$ in Replay-Mobile and Replay-Attack datasets. This remains the same for Rose-Youtu with nearly 
Table 2. The HTER results of class-Specific approaches vs. classindependent ones

\begin{tabular}{|c|c|c|c||c|c|c|}
\hline & \multicolumn{3}{|c||}{ Class-Specific } & \multicolumn{3}{c|}{ Class-Independent } \\
\cline { 2 - 7 } & MAH & GMM & SVM & MAH & GMM & SVM \\
\hline Replay-Mobile & 13.57 & $\mathbf{1 3 . 1 4}$ & 13.90 & 16.57 & 18.85 & 16.99 \\
\hline Replay-Attack & 2.39 & $\mathbf{2 . 1 9}$ & 6.23 & 12.75 & 9.96 & 24.21 \\
\hline Rose-Youtu & 13.24 & $\mathbf{1 1 . 7 3}$ & 18.24 & 18.26 & 17.54 & 22.57 \\
\hline
\end{tabular}

Table 3. Comparison between the proposed ensemble solutions and single best classifier in terms of HTER for each dataset.

\begin{tabular}{|c|c|c|c|}
\hline & Single Best CS & Simple Averaging & Weighted Averaging \\
\hline Replay-Mobile & 13.14 & 12.19 & $\mathbf{9 . 9 5}$ \\
\hline Replay-Attack & 2.49 & 1.57 & $\mathbf{1 . 4 3}$ \\
\hline Rose-Youtu & 11.73 & 11.21 & $\mathbf{9 . 3 0}$ \\
\hline
\end{tabular}

Table 4. Comparison between the proposed weighted averaging ensemble and other approaches in terms of HTER for each dataset.

\begin{tabular}{|c|c|c|c|}
\hline & Weighted Averaging & \multicolumn{2}{c|}{ Other Approaches } \\
\hline Replay-Mobile & $\mathbf{9 . 9 5}$ & $10.40[1]$ & $17.20[7]$ \\
\hline Replay-Attack & 1.43 & $3.13[28]$ & $\mathbf{1 . 2 4}[14]$ \\
\hline Rose-Youtu & $\mathbf{9 . 3}$ & $26.6[23]$ & $16.40[6]$ \\
\hline
\end{tabular}

0.5\% improvement in HTER. Similarly, for the proposed weighted averaging, HTERs of Replay-Mobile and RoseYoutu improve by more than $3 \%$ and $2 \%$ respectively while Replay-Attack gains nearly $1 \%$ improvement.

As seen in Table 4, to compare our best solution, weighted averaging, with the state of the art methods using two-class classification schemes, HTER of $9.95 \%$ in Replay-Mobile dataset is better than $10.40 \%$ in [1] and $17.20 \%$ in [7]. For the Replay-Attack dataset, our HTER of $1.43 \%$ is better than $3.13 \%$ in [28] and slightly worse than 1.24 in [14]. In Rose-Youtu dataset, HTER of $9.30 \%$ is by far better than $26.6 \%$ in [23] and $16.4 \%$ in [6]. It cannot be overemphasised that spoof samples are used only during training to determine the optimal weights of the fusion rule in eq. (4). The spoofing detector design for new clients in the practical use case is purely based on real data only.

In summary, we show that the fusion of different regions, classifiers, and networks can boost the performance of face spoofing detection in all experimental datasets. In addition, class-specific approaches perform consistently better than class-independent methods in benchmark datasets. The proposed ensemble solution based on GA optimisation produces superior performance, as compared to the individual members. Interestingly, our proposed weighted averaging outperforms conventional multiclass classification systems, confirming the merits of the anomaly detection formulation adopted for our face spoofing detection problem.

\section{Conclusion}

Inspired by the promising performance of ensemble learning and the anomaly based approach to face spoofing detection, we proposed novel ensemble techniques based on simple averaging and weighted averaging to fuse one- class classifiers. To exploit the benefits of anomaly classifiers learning using only normal data, we introduced a novel normalisation method to combine the outputs of one-class classifiers trained exclusively using genuine access data. Motivated by the benefits of the subject-specific solutions to the face spoofing detection, we proposed an extension of the client-specific one-class classifiers involving subjectspecific thresholds to capitalise on their ability to be the members of an ensemble learner. To create a pool of spoofing detectors, seven regions of face, three one-class classifiers, and the representations of three CNNs are utilised to constitute 63 different spoofing detector designs. Extensive experiments involving three spoofing datasets confirmed the merits of the client-specific one-class classifier fusion. The experiments were conducted using the existing protocols for the Replay-Mobile and Replay-Attack datasets as well as our new proposed protocol for the Rose-Youtu dataset. The reported results demonstrate that the proposed model yields promising performance compared to the class-independent formulation as well as to conventional multiclass classification models.

\section{References}

[1] A. Anjos and S. Marcel. Counter-measures to photo attacks in face recognition: A public database and a baseline. In 2011 International Joint Conference on Biometrics (IJCB), pages 1-7, Oct 2011.

[2] S. R. Arashloo and J. Kittler. Class-specific kernel fusion of multiple descriptors for face verification using multiscale binarised statistical image features. Trans. Info. For. Sec., 9(12):2100-2109, Dec. 2014.

[3] S. R. Arashloo, J. Kittler, and W. Christmas. An anomaly detection approach to face spoofing detection: A new formulation and evaluation protocol. IEEE Access, 5:13868-13882, 2017.

[4] D. B. Araya, K. Grolinger, H. F. ElYamany, M. A. Capretz, and G. Bitsuamlak. An ensemble learning framework for anomaly detection in building energy consumption. Energy and Buildings, 144:191 - 206, 2017.

[5] C. Bergamini, L. Oliveira, A. Koerich, and R. Sabourin. Combining different biometric traits with one-class classification. Signal Processing, 89(11):2117 - 2127, 2009.

[6] Z. Boulkenafet, J. Komulainen, and A. Hadid. Face spoofing detection using colour texture analysis. IEEE Transactions on Information Forensics and Security, 11(8):18181830, Aug 2016.

[7] I. Chingovska, A. Anjos, and S. Marcel. On the effectiveness of local binary patterns in face anti-spoofing. In 2012 BIOSIG - Proceedings of the International Conference of Biometrics Special Interest Group (BIOSIG), pages 1-7, Sept 2012.

[8] I. Chingovska and A. R. dos Anjos. On the use of client identity information for face antispoofing. IEEE Transactions on Information Forensics and Security, 10(4):787-796, April 2015. 
[9] C. chung Chang and C.-J. Lin. Libsvm: a library for support vector machines, 2001.

[10] A. Costa-Pazo, S. Bhattacharjee, E. Vazquez-Fernandez, and S. Marcel. The replay-mobile face presentation-attack database. In Proceedings of the International Conference on Biometrics Special Interests Group (BioSIG), Sept. 2016.

[11] A. P. Dempster, N. M. Laird, and D. B. Rubin. Maximum likelihood from incomplete data via the em algorithm. Journal of the Royal Statistical Society. Series B (Methodological), 39(1):1-38, 1977.

[12] V. Di Gesù and G. Lo Bosco. Combining one class fuzzy knn's. In F. Masulli, S. Mitra, and G. Pasi, editors, Applications of Fuzzy Sets Theory, pages 152-160, Berlin, Heidelberg, 2007. Springer Berlin Heidelberg.

[13] S. M. Erfani, S. Rajasegarar, S. Karunasekera, and C. Leckie. High-dimensional and large-scale anomaly detection using a linear one-class svm with deep learning. Pattern Recognition, 58:121 - 134, 2016.

[14] M. Farmanbar and Ö. Toygar. Spoof detection on face and palmprint biometrics. Signal, Image and Video Processing, 11(7):1253-1260, Oct 2017.

[15] S. Fatemifar, S. R. Arashloo, M. Awais, and J. Kittler. Spoofing attack detection by anomaly detection. IEEE International Conference on Acoustics, Speech, and Signal Processing (ICASSP), 2019.

[16] J. Galbally, S. Marcel, and J. Fierrez. Image quality assessment for fake biometric detection: Application to iris, fingerprint, and face recognition. IEEE Transactions on Image Processing, 23(2):710-724, Feb 2014.

[17] K. He, X. Zhang, S. Ren, and J. Sun. Deep residual learning for image recognition. CoRR, abs/1512.03385, 2015.

[18] J. Kannala and E. Rahtu. Bsif: Binarized statistical image features. In Proceedings of the 21 st International Conference on Pattern Recognition (ICPR2012), pages 1363-1366, Nov 2012.

[19] B. Krawczyk. One-class classifier ensemble pruning and weighting with firefly algorithm. Neurocomputing, 150:490 - 500, 2015. Special Issue on Information Processing and Machine Learning for Applications of Engineering Solving Complex Machine Learning Problems with Ensemble Methods Visual Analytics using Multidimensional Projections.

[20] C. Lai, D. M. J. Tax, R. P. W. Duin, E. Pekalska, and P. Paclík. On combining one-class classifiers for image database retrieval. In F. Roli and J. Kittler, editors, Multiple Classifier Systems, pages 212-221, Berlin, Heidelberg, 2002. Springer Berlin Heidelberg.

[21] H. Li, W. Li, H. Cao, S. Wang, F. Huang, and A. C. Kot. Unsupervised domain adaptation for face anti-spoofing. IEEE Transactions on Information Forensics and Security, 13(7):1794-1809, July 2018.

[22] L. Li, Z. Xia, L. Li, X. Jiang, X. Feng, and F. Roli. Face antispoofing via hybrid convolutional neural network. In 2017 International Conference on the Frontiers and Advances in Data Science (FADS), pages 120-124, Oct 2017.

[23] W. Li, L. Chen, D. Xu, and L. V. Gool. Visual recognition in rgb images and videos by learning from rgb-d data. IEEE Transactions on Pattern Analysis and Machine Intelligence, 40(8):2030-2036, Aug 2018.
[24] Y. Liu, A. Jourabloo, and X. Liu. Learning deep models for face anti-spoofing: Binary or auxiliary supervision. CoRR, abs/1803.11097, 2018.

[25] L. Nanni. Experimental comparison of one-class classifiers for online signature verification. Neurocomputing, 69(7):869 -873, 2006. New Issues in Neurocomputing: 13th European Symposium on Artificial Neural Networks.

[26] T. Ojala, M. Pietikainen, and T. Maenpaa. Multiresolution gray-scale and rotation invariant texture classification with local binary patterns. IEEE Transactions on Pattern Analysis and Machine Intelligence, 24(7):971-987, July 2002.

[27] V. Ojansivu and J. Heikkilä. Blur insensitive texture classification using local phase quantization. In A. Elmoataz, O. Lezoray, F. Nouboud, and D. Mammass, editors, Image and Signal Processing, pages 236-243, Berlin, Heidelberg, 2008. Springer Berlin Heidelberg.

[28] F. Peng, L. Qin, and M. Long. Face presentation attack detection using guided scale texture. Multimedia Tools and Applications, 77(7):8883-8909, Apr 2018.

[29] M. Sajjad, S. Khan, T. Hussain, K. Muhammad, A. K. Sangaiah, A. Castiglione, C. Esposito, and S. W. Baik. Cnnbased anti-spoofing two-tier multi-factor authentication system. Pattern Recognition Letters, 2018.

[30] M. P. Segundo, L. Silva, O. R. P. Bellon, and C. C. Queirolo. Automatic face segmentation and facial landmark detection in range images. IEEE Transactions on Systems, Man, and Cybernetics, Part B (Cybernetics), 40(5):1319-1330, Oct 2010.

[31] K. Simonyan and A. Zisserman. Very deep convolutional networks for large-scale image recognition. CoRR, abs/1409.1556, 2014.

[32] C. Szegedy, W. Liu, Y. Jia, P. Sermanet, S. Reed, D. Anguelov, D. Erhan, V. Vanhoucke, and A. Rabinovich. Going deeper with convolutions. In 2015 IEEE Conference on Computer Vision and Pattern Recognition (CVPR), pages 1-9, June 2015.

[33] M. Szwoch and P. Pieniażek. Eye blink based detection of liveness in biometric authentication systems using conditional random fields. In L. Bolc, R. Tadeusiewicz, L. J. Chmielewski, and K. Wojciechowski, editors, Computer Vision and Graphics, pages 669-676, Berlin, Heidelberg, 2012. Springer Berlin Heidelberg.

[34] D. M. Tax and R. P. Duin. Support vector data description. Machine Learning, 54(1):45-66, Jan 2004.

[35] D. M. J. Tax and R. P. W. Duin. Combining one-class classifiers. In Proceedings of the Second International Workshop on Multiple Classifier Systems, MCS '01, pages 299-308, London, UK, UK, 2001. Springer-Verlag.

[36] P. Viola and M. Jones. Rapid object detection using a boosted cascade of simple features. In Proceedings of the 2001 IEEE Computer Society Conference on Computer Vision and Pattern Recognition. CVPR 2001, volume 1, pages I-I, Dec 2001.

[37] V. Štruc and N. Pavešić. Photometric normalization techniques for illumination invariance, pages 279-300. IGIGlobal, 2011.

[38] J. Yang, Z. Lei, and S. Z. Li. Learn convolutional neural network for face anti-spoofing. CoRR, abs/1408.5601, 2014. 